\title{
CIRSE and World Interventional Radiology
}

\author{
Robert Morgan ${ }^{1}$ Birgit Slijepcevic ${ }^{2}$ \\ ${ }^{1}$ Department of Radiology, St. George's Hospital, London, United \\ Kingdom \\ ${ }^{2}$ Cardiovascular and Interventional Radiological Society of Europe, \\ Vienna, Austria \\ J Clin Interv Radiol ISVIR 2018;2:83-86
}

\begin{abstract}
Address for correspondence Robert Morgan, MB ChB, MRCP, FRCR, EBIR, FCIRSE, Department of Radiology, St George's Hospital, London SW17 0QT, United Kingdom (e-mail: robert.morgan@ stgeorges.nhs.uk).
\end{abstract}

\section{Introduction}

The Cardiovascular and Interventional Radiological Society of Europe (CIRSE) is a nonprofit scientific and educational society that was founded in 1985 with the aim to "improve patient care through the support of teaching, science, research, and clinical practice in the field of cardiovascular and interventional radiology." It currently has more than 32,000 health care professionals, including 7,000 academic members. In terms of global outreach, CIRSE membership covers a total of 38 European and international IR societies and 27 corporate members.

The exponential growth in CIRSE membership over the past decades gives an indication of the importance that interventional radiology (IR) is gaining worldwide as a very dynamic and ever-expanding discipline. The benefits of IR therapies have been demonstrated in several studies.

The future-oriented and minimally invasive therapies IR offers have become attractive alternatives to more invasive treatments and have led to an increased patient demand. Also, lower morbidity and mortality rates and better quality of life have been associated with IR therapies compared with surgically invasive alternatives. Cost-effectiveness is another important benefit of many IR therapies due to faster recovery periods, which allows for shorter hospital stays and a faster return to the labor market. From an IR's perspective, the advantages of IR have long been very clear, despite the reluctance of other specialties to recognize these.

However, to strengthen IR in the long term, those involved also need to be aware of the risks and challenges that this comparatively young discipline is facing and actively discuss and address these. Competition from other specialties, lack of clinical control, poor recognition in undergraduate education, and unawareness or limited recognition of IR by the public and health service managers remain key issues. Addressing these in a comprehensive fashion will help IR consolidate its position and also demonstrate the advantages of minimally invasive therapies to other medical disciplines, relevant patient groups and referrers.

Representing the European and large parts of the international IR community, the main mission of CIRSE is to develop activities and initiatives that support IR throughout the entire spectrum: from attracting and training young interventional radiologists (IRs), to proving the highest quality level of continuing education and training, to providing internationally recognized certification and research projects as well as peer-reviewed journals that will further expand the evidence-base upon which IR is built.

In the following sections, four areas of CIRSE's activities in these fields are discussed: (1) education and IR's next generation, (2) certification, (3) research and guidelines, and (4) communication and global collaboration. received

May 11, 2018

accepted after revision

May 14, 2018

published online

July 31, 2018
DOI https://doi.org/

10.1055/s-0038-1668605.

ISSN 2457-0214.
Copyright (C2018 by Indian Society

of Vascular and Interventional

Radiology
License terms

()(1) $\odot \circledast$ 


\section{Education}

The provision of unbiased scientific education is of paramount importance for CIRSE, as it will enhance the credibility, ensure high-quality standards, and patient safety.

CIRSE annually organizes the world's largest international IR meeting, which attracts almost 7,000 participants from 85 countries. The CIRSE Annual Meeting and Postgraduate Course covers all areas of IR, including arterial, aortic, and venous interventions; interventional oncology (IO); embolization; neurointerventions; and nonvascular interventional procedures.

With IO quickly establishing itself as the fourth pillar of modern cancer care, ${ }^{2}$ CIRSE organizes the Annual European Conference on Interventional Oncology (ECIO), which has become the prime meeting in this quickly expanding field. More than 1,300 participants from more than 50 countries attend this meeting every year, and the strong focus on the interdisciplinarity and the internationally renowned faculty are strong factors in the continuous growth and importance of the meeting on a global scale.

Beyond these two major conferences, CIRSE biennially organizes the International Conference on Complications in Interventional Radiology and offers four to five advanced, practice-oriented clinical procedure trainings per year. Attesting CIRSE's unbiased scientific approach, all live educational events are accredited by the European Accreditation Council for Continuing Medical Education (EACCME).

In the interest of also providing high-quality content to CIRSE's international members, live and on-demand services are available for almost all events, and thereafter accessible via European Board of Interventional Radiology (EBIR) online. In the near future, the Society's online portfolio will be further upgraded with the introduction of the CIRSE Academy, regrouping peer-reviewed learning materials, including videos and self-assessment quizzes corresponding to topics in the European Curriculum and Syllabus for IR, and the CIRSE library, which will contain all presentations from past congresses.

\section{Involving the Next Generation}

With the rapid expansion of and increasing demand for IR treatments, the availability of well-trained IR practitioners needs to be ensured in the long term. One important reason for the growing IR staff shortage in Europe is the lack of awareness for this discipline among undergraduate students, as pointed out by Lee and Lee. ${ }^{3}$

Therefore, CIRSE launched a program to engage with medical students already in 2011. The "be inspIRed" CIRSE student program aims to attract and involve the next generation of IRs and allows medical students to attend the CIRSE and ECIO congresses free of charge. Approximately 1,300 students participated in the Student Program to date, and regular surveys confirm the positive effect this initiative has on building upon the awareness of and interest in IR among participants. ${ }^{4}$ An Interventional Radiology Curriculum for Medical Students was published in 2012 and is currently being revised, with the aim of addressing the lack of awareness and providing medical students with basic knowledge of IR.

More recently, the European Trainee Forum was created to guide young professionals in their career and provide an international forum for exchange and cooperation.

Starting from a solid foundation of clinical knowledge and practical skills, the next generation of IRs will certainly be able to uphold IR's pioneering role in modern medicine and

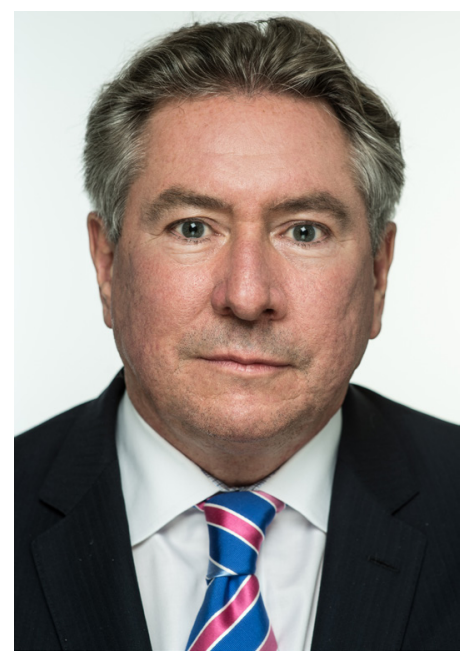

Robert Morgan, MB ChB, MRCP, FRCR, EBIR, FCIRSE ensure the continuous supply of services while further developing cutting-edge techniques and related technologies. CIRSE therefore takes a very active approach toward educating, supporting, and, as outlined below, certifying qualified young specialists.

\section{Certification}

As pointed out by Tsetis et al, "the development and definition of core IR skills, delivered by training and accredited by summative assessment is a critical step for optimal service delivery and [...] a vital step in maintaining patient safety and ensuring high-quality standards." ${ }^{5}$ The European Curriculum and Syllabus for Interventional Radiology and the European Board of Interventional Radiology (EBIR) were established for this purpose and were widely endorsed by national and regional IR societies. ${ }^{6}$ The EBIR has been running successfully for 8 years and has accredited more than 526 EBIR holders from 36 countries to date. IRs at the beginning of their career are strongly encouraged to sit the exam, but also more experienced professionals can greatly benefit from certifying their competence. The constantly increasing demand and interest confirm the global recognition and value of this certification of IR knowledge and skills. EBIR is endorsed by the European Society of Radiology and the European Union of Medical Specialists.

For the establishment of internationally recognized, independent certification and accreditation processes in IR, it is crucial that they allow experts and departments to demonstrate their competence. In the rapidly growing field of IO, CIRSE is supporting the establishment of high-quality IO services with the soon-to-be-launched International Accreditation Scheme for Interventional Oncology Services (IASIOS). ${ }^{7}$ With this project, CIRSE has developed a partnership-based approach that will allow centers to apply for accreditation and go through an auditing and assessment process of the center's available infrastructure-a prerequisite for safe practice and high-quality services.

In parallel, CIRSE is developing certification and accreditation activities for endovascular specialists and services 
that will support IRs performing endovascular therapies. High standards of service provision and quality assurance, as outlined in the CIRSE recommendations for the Provision of Interventional Radiology Services in Europe, ${ }^{5}$ and the continuous improvement of IR's evidence base and standards, remain key areas for ensuring a bright future for IR.

\section{Research and Guidelines}

With its privileged access to world-leading experts in IR, CIRSE biennially elects a Standards of Practice Committee in charge of drafting and updating standards for IR practice. Total 35 guidelines on a large array of topics have been published since 2003, often in collaboration with national IR societies. Involvement in the drafting of multidisciplinary national and international guidelines, such as the European Cancer Society's (ECCO) Essential Requirements for Quality Cancer Care series, will further enhance IR's standing in different areas.

Beyond standard setting, CIRSE more recently started to engage in its own clinical research activities as an independent scientific sponsor. Since 2013, the Society has built up an in-house research infrastructure to conduct high-quality observational studies, adhering to a set of quality principles and the ISO 14155 standard on clinical investigation of medical devices for human subjects. While recognizing that clinical trials still represent the gold standard in terms of clinical data collection, the merits of scientifically sound observational studies are nowadays widely recognized. This particularly holds true for IR, as for ethical but also logistic and political reasons, it has often proven difficult to conduct large-scale clinical trials in IR. Hence, CIRSE aims to further the collection of high-quality, real-life data that will demonstrate not only the safety and effectiveness of IR therapies but also show their mid- to long-term benefits in terms of health economics and quality of life.

The CIRSE Registry for SIR-Spheres Therapy (CIRT) is the Society's flagship research project with the primary objective being to observe the real-life clinical application of selective internal radiation therapy (SIRT) as well as the impact of the treatment in clinical practice. The project enrolled more than 1,000 patients in 2 years, and the 2-year follow-up period is currently ongoing. In parallel, CIRSE also sponsors a regulatory study to observe the real-life clinical application of radioembolization with SIR-Spheres in France (CIRT-France), which enrolled its first patient in late 2017.

More recently, the CIRSE Registry for LifePearl Microspheres (CIREL) was launched with the primary objective to categorize observed usages of transarterial chemoembolization (TACE). With several new projects in the pipeline, CIRSE is hopeful to make a significant contribution to the evidence base supporting IR.

\section{Communication and Collaboration}

Last but not least, the long-term success of IR will be influenced by the discipline's capacity to effectively communicate what IR therapies can do for patients. Communication needs to target different audiences and convey tailored messages backed up by standards, certification and quality assurance systems, and clinical data. CIRSE has established a wide portfolio of communication channels as well as a global network of IR societies to help make IR more prominent through group membership.

In the framework of its group membership, CIRSE has established a unique network of national and regional IR societies, spanning throughout Europe and across the globe from Canada and South America, to India, Japan, and Australasia. In this system, all national IR societies join on an equal footing with the opportunity for each of their members to gain profit from all CIRSE member services and offered benefits CIRSE's group membership system that also allows for regular exchange and annual liaison meetings at the leadership level to address challenges IR is facing globally and to develop collaborative strategies to successfully overcome these and strengthen IR worldwide.

Out of CIRSE's 38 group members, 21 have also endorsed Cardiovascular and Interventional Radiology (CVIR) as their official organ and actively contribute to the success of the journal. The double-blind peer-reviewed journal has been publishing original research work in IR since 1987. In 2017, CVIR Endovascular was also launched as a multidisciplinary, peer-reviewed open-access addition to CIRSE's publication family in the field of endovascular interventions.

In terms of patient outreach, dedicated material informing patients about the benefits of IR therapies are produced and regularly updated by CIRSE. A dedicated task force is currently updating CIRSE's Patient Information Brochures, which explain the most common IR interventions to the general public. With the help of such publications as well as local initiatives involving patient groups, CIRSE aims to create more awareness for the alternatives IR can offer in the treatment of many different medical conditions.

\section{Conclusion}

Interventional radiology has seen a tremendous expansion over the past decades and keeps growing globally. While this development is very encouraging, several obstacles need to be tackled to ensure a bright future. CIRSE and likeminded national and regional IR societies play a crucial role in addressing these challenges in a coordinated manner by

- Providing unbiased (continuing) education and clear training pathways and supporting the next generation of IR

- Offering an internationally recognized certification in IR as well as accreditation opportunities for specialized IR services

- Encouraging clinical research and standards for procedures as well as infrastructure and IR service provision

- Creating awareness among other specialties, national health services, patients, and the general public.

\section{Conflict of Interest}

None. 


\section{References}

1 Articles of Association of the Cardiovascular and Interventional Radiological Society of Europe. www.cirse.org. Accessed April 9, 2018

2 Kim HS, Chapiro J, Geschwind JH. From the Guest Editor: Interventional Oncology: the fourth pillar of oncology. Cancer J 2016;22(6):363-364

3 Lee AM, Lee MJ. Teaching IR to medical students: a call to action. Cardiovasc Intervent Radiol 2018;41(2):203-205

4 CIRSE Student Programme. www.cirse.org/students. Accessed on April 9, 2018

5 Tsetis D, Uberoi R, Fanelli F, et al. The Provision of Interventional Radiology Services in Europe: CIRSE recommendations. Cardiovasc Intervent Radiol 2016;39(4):500-506

6 European Curriculum and Syllabus for IR. www.cirse.org/curriculum_syllabus. Accessed on April 9, 2018

7 International Accreditation System for Interventional Oncology Services (IASIOS). www.iasios.org. Accessed April 25, 2018

8 CIRSE Registry for SIR-Spheres Therapy (CIRT). www.cirse.org/ cirt. Accessed on April 9, 2018

9 CIRSE Registry for LifePearl Microspheres (CIREL). www.cirse. org/cirel. Accessed April 9, 2018

\section{Suggested Readings}

1 Bezzi M, Brountzos E, Hausegger K, et al. European curriculum and syllabus for interventional radiology. Eur Curric Syllabus Interv Radiol CIRSE: ISBN: 978-3-9502501-3-8

2 Helmberger T, Martí-Bonmatí L, Pereira P, et al. Radiologists' leading position in image-guided therapy. Insights Imaging 2013;4(1):1-7

3 Kim HS, Chapiro J, Geschwind JH. From the Guest Editor: Interventional Oncology: the fourth pillar of oncology. Cancer J 2016;22(6):363-364

4 Kaufman JA, Reekers JA, Burnes JP, et al. Global statement defining interventional radiology. J Vasc Interv Radiol 2010;21(8):1147-1149

5 Lee AM, Lee MJ. Teaching IR to medical students: a call to action. Cardiovasc Intervent Radiol 2018;41(2):203-205

6 Lee MJ, Belli AM, Brountzos E, Morgan R, Reekers JA. Specialty status for interventional radiology: the time is now. Cardiovasc Intervent Radiol 2014;37(4):862

7 Tsetis D, Uberoi R, Fanelli F, et al. The Provision of Interventional Radiology Services in Europe: CIRSE recommendations. Cardiovasc Intervent Radiol 2016;39(4):500-506 